\title{
Introducing a Group Therapy Program (PLAN D) for Young Outpatients with Derealization and Depersonalization: A Pilot Study
}

\author{
Rahel Flückiger ${ }^{\mathrm{a}}$ Stefanie J. Schmidt ${ }^{\mathrm{b}} \quad$ Chantal Michel $^{\mathrm{a}} \quad$ Jochen Kindler $^{\mathrm{a}}$ \\ Michael Kaess ${ }^{a, c}$ \\ aUniversity Hospital of Child and Adolescent Psychiatry and Psychotherapy, University of Bern, Bern, Switzerland; \\ ${ }^{b}$ Department of Clinical Psychology and Psychotherapy, University of Bern, Bern, Switzerland; ' ${ }^{C}$ Clinic for Child and \\ Adolescent Psychiatry, Centre for Psychosocial Medicine, University of Heidelberg, Heidelberg, Germany
}

\section{Keywords}

Derealization · Depersonalization · Cognitive behavioral therapy · Group intervention · Outpatients

\begin{abstract}
Depersonalization and derealization (DD) cause significant distress and are associated with poor role and social functional outcomes. Despite the relatively high prevalence of DD symptoms and the chronic course in those suffering from a DD disorder, there still exists a need for effective interventions. Preliminary evidence indicates that cognitive behavioral therapy (CBT) delivered in an individual setting demonstrates some positive intervention effects for patients with DD regarding their symptom levels. By considering DD-specific treatment needs, a group therapy program was developed as an add-on therapy based on CBT techniques called PLAN D comprising the following elements: psychoeducation, lifestyle interventions, acceptance and mindfulness training, and new patterns of DD-related cognitions. In a pilot study, we present an 8-week group intervention for adolescents and young adults with DD disorder. To our knowledge, no standardized group intervention program for DD exists so far. Thus, this novel intervention represents a promising opportunity to positively influence long-term outcomes and course of DD.
\end{abstract}

(C) 2021 The Author(s).

Published by S. Karger AG, Basel

\section{Introduction}

"I feel like I am watching my life from behind glass, as if I am living in a dream" - this is a typical statement from someone experiencing derealization and/or depersonalization (DD). DD is a perceptional reaction and psychological phenomenon, especially occurring when individuals are highly distressed, traumatized, very tired, anxious, or intoxicated [1]. In the Diagnostic and Statistical Manual of Mental Disorders, Fifth Edition (DSM-5) [2], clinically relevant DD is diagnosed as depersonalization/ derealization disorder in the chapter of dissociative disorders. Therein, it is defined as a persistent or recurrent experience of unreality and detachment from oneself or their surroundings, while reality testing remains intact and the symptoms cause clinically significant distress or impairment in functioning [2].

Prevalence rates of $1.2-2.4 \%$ were found for clinically significant DD symptoms in the community and of $30-$ $82 \%$ in clinical samples, in a systematic review [3]. Furthermore, DD (disorder) often exists as a comorbidity, especially in depression and anxiety [4]. Most DD experiences are transient and disappear as situational factors ease. However, in a relevant minority of cases, DD persists for days, weeks, or months, reoccurs, or remains permanent [3]. Age of onset is adolescence, and an earlier onset is associated with higher severity and poorer prog- karger@karger.com www.karger.com/psp

Karger $\stackrel{\text { ' }}{5}$

BOPEN ACCESS
(C) 2021 The Author(s)

Published by S. Karger AG, Basel

This is an Open Access article licensed under the Creative Commons Attribution-NonCommercial-4.0 International License (CC BY-NC) (http://www.karger.com/Services/OpenAccessLicense), applicable to the online version of the article only. Usage and distribution for commercial purposes requires written permission.
Correspondence to:

Rahel Flückiger, rahel.flueckiger@upd.unibe.ch 


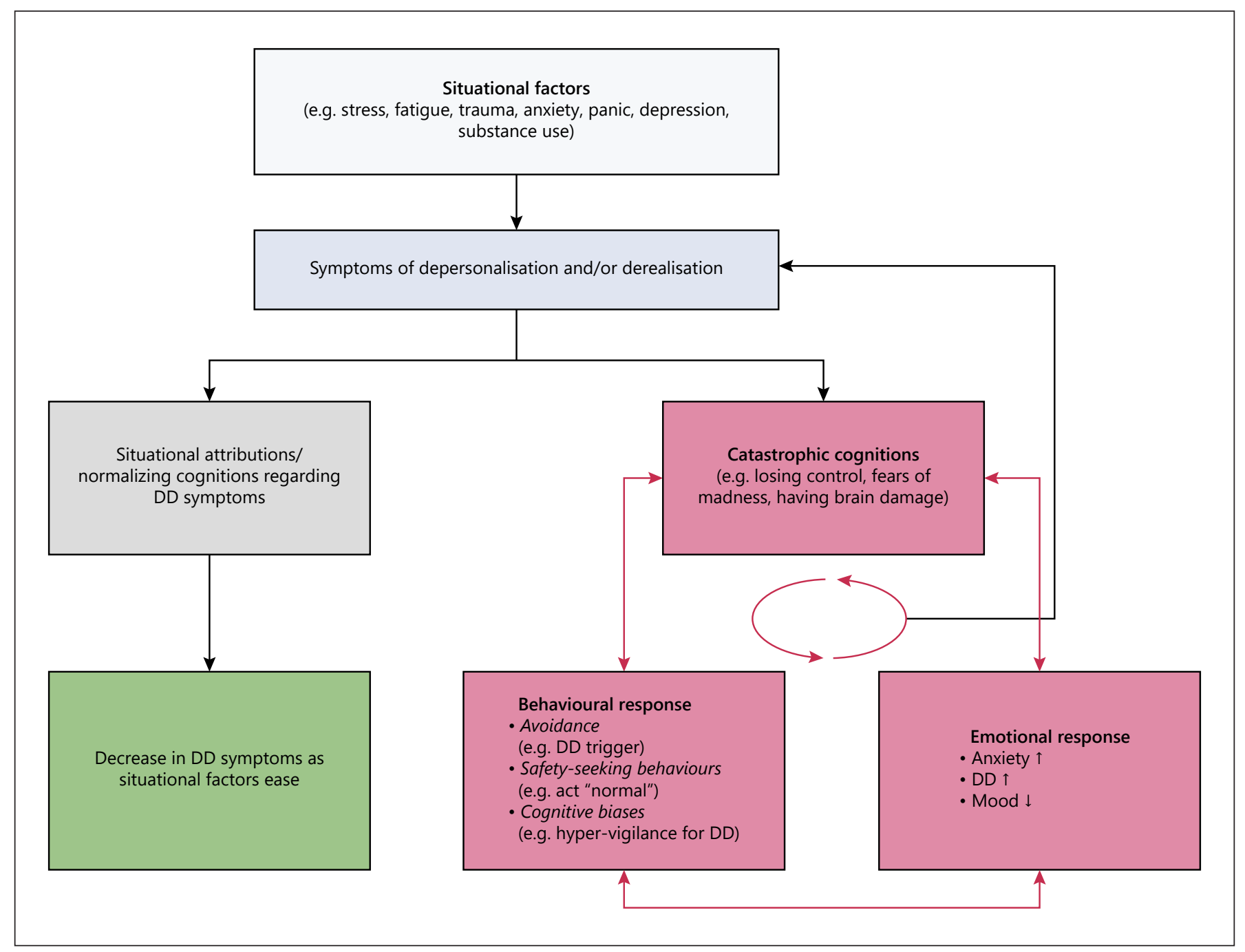

Fig. 1. Cognitive behavioral model of DD adapted from Hunter [14] explaining development and maintenance of DD disorder. DD, derealization/depersonalization.

nosis $[4,5]$. Individuals with DD frequently are distressed, worry about their mental state, and are frightened of becoming crazy, a common fear not only in patients who experience DD but also in patients experiencing ultrahigh-risk (UHR) symptoms. Furthermore, chronic DD often is associated with functional impairments $[1,6,7]$. Apart from an isolated DD disorder or comorbid phenomena, DD commonly appears in subjects at clinical high risk (CHR) for psychosis. Thus derealization, but not depersonalization, is one of the 14 basic symptoms that were shown to be specific to the development of firstepisode psychosis [8]; basic symptoms defined as self-experienced disturbance of "normal" mental processes [9, 10] are employed in 2 CHR criteria [11-13]: cognitive disturbances (COGDIS) and cognitive-perceptive (COP-
ER) basic symptoms. They are part of the 2 complementary early detection approaches to the characterization of the CHR state of psychoses: the UHR and the basic symptoms criteria [13]. The (attenuated) positive symptoms as part of the UHR criteria are distinct from the basic symptoms since they are experienced by the individual - depending on the insight into its abnormal nature - as real, normal thinking, and feeling $[9,10]$. Derealization as part of the COPER criterion is rated as below attenuated positive symptom level (for more details to this distinction, see [10]). Taken together, the high prevalence of DD symptoms, its role as a psychosis risk symptom (according to basic symptoms), and the chronic course in those suffering from a DD disorder highlight the need for effective targeted interventions. 
Therefore, Hunter et al. [1, 6] conceptualized a cognitive behavioral model for the development and maintenance of DD (Fig. 1) based on etiological models for anxiety disorders (e.g., panic, health anxiety). The model suggests that chronic DD may result from catastrophic interpretation of common, normally transient symptoms of DD as being indicative of a serious mental disorder. This leads to an exacerbation and perpetuation of DD symptoms through the development of cognitive biases and behaviors forming a maintenance cycle $[1,14]$. Although the cognitions are disorder specific, the similarity between anxiety disorder and DD lies in this misinterpretation [6, 15]. Accordingly, Hunter et al. [6] modified cognitive behavioral therapy (CBT) for anxiety disorders to $\mathrm{DD}$, respectively, for their specific thought contents which resemble a "mental health anxiety." They created a CBT protocol in 3 phases with the following interventions: (1) psychoeducation and normalizing and diary keeping, (2) reducing avoidance and safety behavior as well as self-focused attention and symptom monitoring, and (3) focusing on maintaining progress and relapse. They investigated this CBT protocol in an open trial investigating a sample of 21 patients with $\mathrm{DD}$ disorder (mean age 38 years) and with a mean duration of 14 years of DD. In an open 2-year study, the intervention was carried out with a mean number of 13 sessions. After intervention, $29 \%$ of the participants no longer met DD criteria. While they found a significant reduction in clinicianrated DD symptoms, to our knowledge no replication study currently exists.

Considering the paucity of literature on psychological interventions in DD, there are several specific interventions considered to be successful in patients with DD: DD assessment and exploration of relevant factors which enhance and reduce DD symptoms, psychoeducation, training of grounding strategies or mindfulness, lifestyle interventions (e.g., sleep, substance use, activity), reducing avoidance, safety behavior, symptom monitoring and self-focused attention, cognitive restructuring techniques to challenge negative automatic DD-related cognitions, techniques to facilitate the controlled re-experience of emotions as well as targeting the high arousal and anxiety level $[5,7,14,16-19]$. An important clinical feature in DD patients is the often-reported emotional detachment. It has been shown that chronic DD disorder patients showed different skin conductance response to emotional stimuli compared to healthy or clinical controls meaning a reduced autonomic response to unpleasant stimuli, suggesting a selective inhibitory mechanism on emotional processing [20]. A replication study with DD patients suggested that the cognitive evaluation of emotional stimuli is disconnected from their bodily or autonomic response, respectively [21], which reflects an increased introversion and control of emotional impulses. In the same study, mindfulness exercises were found to be helpful in modulating this autonomic response since they immediately decreased DD intensity [21]. In sum, primarily CBT elements with a special focus on DD-specific cognitions, enriched by emotion regulation techniques such as mindfulness and relaxation training, are applied and conferred to be helpful in DD. Additionally, acceptance and commitment therapy (ACT) [22] is also discussed as a useful element in DD, especially because ACT targets experiential avoidance, which is assumed in dissociative conditions such as DD [23]. Despite this detailed DD conceptualization and DD-specific knowledge, to date, no standardized treatment protocol for DD has been established, and the need for (novel) therapeutic approaches is still ongoing. To address this, we report on the development and implementation of a DD-specific group program targeting young patients with DD disorder by giving them a first CBT-based intervention.

\section{Materials and Methods}

\section{Rationale and Conceptualization of PLAN D}

In the "Bern Early Detection and Intervention Center for Mental Crises" (FETZ Bern), where we apply an indicated prevention approach of psychoses, DD is a frequently reported phenomenon among our help-seeking outpatients (30\% with intermittent DD symptoms, $8 \%$ with DD disorder). Based on our indicated prevention approach, we aimed to develop a structured psychotherapy manual for individuals with DD and to deliver this psychotherapy as a group program, in order to bring DD patients together and foster group processes, which is known to facilitate normalization of experiences and receive early psychological intervention. In the FETZ Bern, the approach of normalizing experiences and fears related to these symptoms is generally used by the FETZ therapists not only in the treatment of DD but also in CBT offered to patients with UHR symptoms, where an evidence-based psychotherapy by van der Gaag et al. [24] is used. Almost every UHR patient fears the loss of control of his/her psyche, and the therapeutic approach in the FETZ Bern aims to emphasize that the patient is not crazy and to create hope for recovery. The program's name "PLAN D" not only encompasses the various elements and intervention techniques (Table 1) but also takes into account that many DD patients often try a lot of, and struggle with, other plans to eliminate DD experiences before seeking help. To that end, PLAN D should present an alternative, more reasonable plan. The group program follows a CBT approach that considers the DD-specific interventions that exist in the current literature by including elements of ACT. PLAN D is provided as an add-on therapy for 4 to 6 adolescent and/or young adult outpatients with clinically relevant DD (disorder). The program comprises 8 weekly sessions of 90 min carried 
Table 1. Elements and techniques of PLAN D

P Psychoeducation (2 sessions)

Aim: information about DD including definition and prevalence; establish an explanatory model (the cognitive behavioral model of DD, see Fig. 1), initiate a DD diary

Techniques: psychoeducation

$\mathrm{L} \quad$ Lifestyle interventions (1 session)

Aim: eliminate maladaptive (safety) behavior and misconceptions about lifestyle behaviors by interactively providing information about lifestyle with a special focus on stress, sleep, substance (ab-)use, social, and physical activity Techniques: psychoeducation, model learning

A Acceptance and mindfulness training (1 session)

Aim: acceptance of DD as well as aversive internal content (e.g., thoughts and feelings) whilst pursuing personal values; learn to be mindful, lessen symptom monitoring, and coping with experiential avoidance Techniques: guided mindfulness training ${ }^{1}$, relaxation, role plays

$\mathrm{N} \quad$ New patterns of DD-related cognitions (3 sessions)

D Aim: identifying and modifying dysfunctional cognitions about DD and demonstrating their impact on emotions and behavior

Techniques: cognitive restructuring techniques and evidence gathering

DD, derealization and/or depersonalization. ${ }^{1}$ Thenceforward, a short mindfulness training is part of every following sessions.

out by 2 therapists. The sessions are accompanied by a "Booklet" with worksheets, such as the DD diary and materials. The sequence of the sessions is structured as follows: beginning with intensive psychoeducation $(\mathrm{P})$ as well as establishing a DD model that can be transferred to every individual case. The diary keeping helps to reveal relevant factors such as situations, emotions, cognitions, and behaviors, which are then discussed in the following lifestyle session (L). The focus of this session lies in correction of false beliefs about DD-related factors, psychoeducation about avoidance behavior, and stress management. In the " $\mathrm{A}$ " session, the focus lies in practicing acceptance as a helpful coping strategy for DD-related emotions and training in mindfulness. From this session on, in every following session, mindfulness or meditation will be exercised at the beginning or end of the sessions. The next 3 sessions ( $\mathrm{N}$ and $\mathrm{D})$ target the reconstruction of DD-specific cognitions. The eighth session is a closing session containing retrospection, summary, feedback, and resource activation in the group. All subjects (or their parents or guardians) had given their written informed consent. The study protocol was approved by the institute's committee on human research.

\section{Study Design}

PLAN D was evaluated in a naturalistic setting in the FETZ Bern with data of 2 uncontrolled groups of 4 outpatients. Patients were recruited after the diagnostics if they fulfilled the following inclusion criteria: $\geq 13$ years and clinically relevant DD disorder according to ICD-10 [25] or DSM-5 [2]. For the recruitment, DD symptoms were assessed in clinical interviews $[11,12]$. As an outcome measure, we evaluated the severity and frequency of DD symptoms according to the "Cambridge Depersonalization Scale" (CDS) [26]. The CDS was used as a pre- and postmeasure at baseline before the therapy and 6 months after baseline. The CDSs were handed out in the first session and sent by post 6 months after as a postmeasure. No further clinical evaluation or diagnostic was done at the end of PLAN D as well as 6 months later.

\section{Study Population}

The studied sample comprised 8 outpatients ( 4 males) between 17 and 23 years ( $M \pm S D: 20.27 \pm 2.01$ years). According to ICD-10 [25], all patients fulfilled criteria of a derealization/depersonalization disorder (F48.1), 4 patients additionally had a current depressive episode (F32.1), one had a schizotypal disorder (F21), and one a bipolar disorder (F31.1), and 6 of them further met criteria for a CHR state for psychosis according to the EPA [13]. Three patients with a depressive episode had current antidepressant medication, and 1 patient with a CHR state and schizotypal disorder had an antipsychotic medication. All medication has been established before the start of PLAN D and was stable during the program. Duration of DD was 35.0 months on average (SD: 21.94, Md: 31.0, range: 7-66; 1 patient reported lifetime duration of DD). Seven patients reported enduring DD, and 1 patient reported episodic DD of at least once in a month during a week. Two patients had no treatment history, 5 had psychological outpatient treatment, and one had a psychiatric inpatient treatment before PLAN D.

\section{Results}

As shown in Figure 2, at the beginning of PLAN D, all CDS scores were above the cutoff $(\geq 65)$ for clinically relevant DD ( \pm SD: $99.14 \pm 50.12$, Md: 82.0, range: 66209). At postmeasure, 3 persons (1 male/2 females) showed clinically relevant DD according to the CDS (M \pm SD: $74.71 \pm 34.99$, Md: 59.0, range: 49-149). Although 


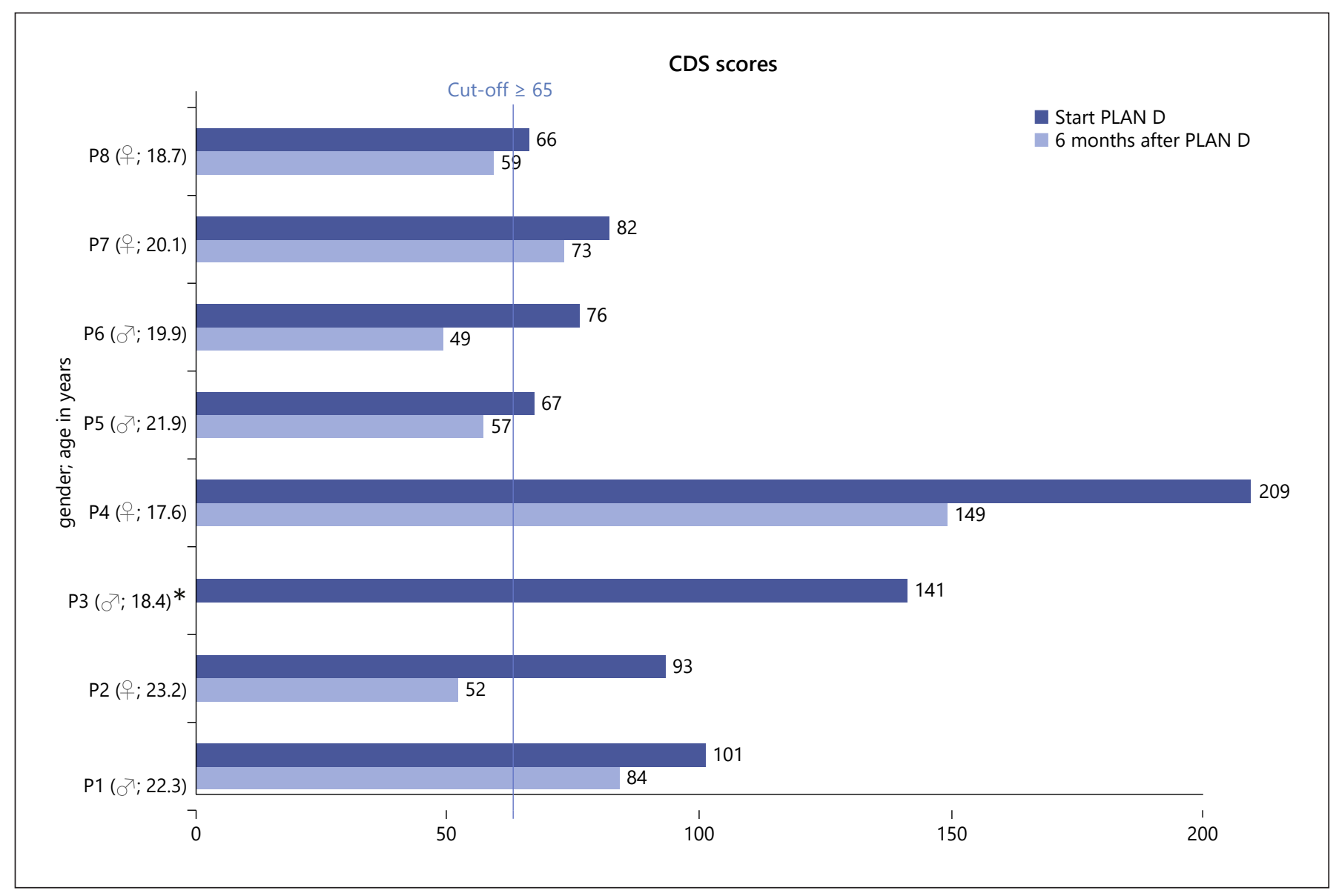

Fig. 2. Pre- and postmeasure scores of the CDS at baseline before the PLAN D group intervention and 6 months after baseline for the sample of 8 participants (P1-P8). P2, 3, and 8 did not continue any therapy after PLAN D; P1 and 4-7 continued further psychological treatment after PLAN D. *Follow-up measurement is missing. CDS, Cambridge Depersonalization Scale.

the effect size of the decrease in DD symptoms was large (Wilcoxon signed rank test: $Z=-2.366, p=0.018$, Rosenthal's $r=0.894$ ), according to Figure 2, the mean reduction in the CDS is about $20 \%$, which reflects a minimal improvement in psychopathology. An informal qualitative assessment showed high acceptance and heterogenic, but overall, very good ratings of the different sessions (best rating for sessions "N and D"; lowest rating for session "L," see Table 1).

\section{Discussion}

According to an existing CBT-based treatment protocol for DD disorder [6], we conceptualized and implemented a group intervention called PLAN D to face the limited availability of disorder-specific interventions for
DD. In our first data, we found a reduction in the overall CDS score 6 months after the start of PLAN D with only 3 persons still being over the clinical cutoff. Although we found a statistically strong effect of the intervention (Rosenthal's $r>0.8$ ), due to small sample size, lack of a control group, and no immediate posttreatment measurement (approximately 6 months after baseline), this finding should be interpreted with some caution as the reduction in psychopathology of $20 \%$ reflects a minimal improvement. According to our informal quality assessment, the acceptance of the group was very good. Our experiences showed that in the recruiting process some patients have been very open and were immediately dedicated to take part in PLAN D while others were more skeptical and initially reluctant. Consequently, we were confronted with the challenge to carefully explore their reservations and to motivate them to confront, 
rather than avoid, specific (social) situations. Therefore, in some cases taking part in PLAN D, group sessions posed a first step in their therapeutic process helping to overcome their (social) withdrawal. As from the first group session on and in the interaction between participants, respectively, we observed a normalizing effect concerning DD experiences. Besides the good compliance, patients showed high interest and involvement especially in the psychoeducation sessions. During these sessions, we carefully elaborate with participants to learn $\mathrm{DD}$ is a possible psychological reaction to stress (according to the model in Fig. 1) as well as also to address the distress about $\mathrm{DD}$ as a potential psychosis risk symptom. In the feedback session, all participating patients reported to meet other DD-affected persons as very helpful as well as get and give each other support while receiving professional $\mathrm{DD}$-specific intervention. Even though the qualitative ratings of each individual session were relatively high, sessions $5-7$ ( $\mathrm{N}$ and $\mathrm{D}$ sessions, see Table 1) were rated highest by participants and in our view highlight the importance of cognitive techniques to cope with DD by overcoming dysfunctional cognitions. In the "lifestyle intervention" session, we discuss existing concerns about lifestyle or behavior and their impact on DD such as sleep, nutrition, or substance use more general and clear up with possible maladaptive beliefs. In this session, participants already demonstrated good knowledge of the topic by sharing their personal experiences with others, which could be the reason for the lowest rating. Besides this, participants found the experience to be positive throughout the course of therapy. However, we are presenting very preliminary data from a naturalistic setting, and some limitations should be noted, such as the lack of a control group and the small sample size. Furthermore, clinical evaluation immediately after completion of the group program PLAN D is not included in the current pilot study, and future studies should endeavor to include such measures. Evaluation of the program as a randomized controlled trial with appropriate control groups would further help to disentangle therapy effects from other possible confounding variables (e.g., other therapies or natural symptomatic reduction) and should be considered in future studies. Nonetheless, PLAN D presents a promising option as an adjunct to existing treatments given in individual settings, as it consists of well-established CBT (based) techniques that can be offered in a time-limited group setting. Furthermore, if our initial findings would be replicated or even improved, it would be considered a cost-effective option for psychological treatment for DD. Future research should replicate these promising results by addressing the named limitations and continually improve interventions for this highly distressing condition. Early diagnosis and psychoeducation help alleviate DD-associated distress and may promote recovery or prevent a long-term DD history and, consequently, a possible psychotic development. To that end, PLAN D represents a novel, brief intervention and a promising opportunity to positively influence the prognosis of patients suffering from DD.

\section{Acknowledgments}

We thank Madelyn Thomson for the English editing. A special thank you goes to our outpatients for their participation in the PLAN D group and all the other following patients to constantly improving our group program.

\section{Statement of Ethics}

All procedures contributing to this work comply with the ethical standards of the relevant national and institutional committees on human experimentation and with the Helsinki Declaration of 1975, as revised in 2013. The Human Research Ethics Committee of the Canton Bern approved the study (ID PB_2016-01991). All participants gave written informed consent, and in minors, parental written informed consent was provided.

\section{Conflict of Interest Statement}

All authors declare no conflicts of interest regarding the topic of this article.

\section{Funding Sources}

The FETZ Bern is a cooperation of the University Hospitals of Psychiatry and Psychotherapy and Child and Adolescent Psychiatry and Psychotherapy, University of Bern and the Soteria Bern. This study was supported by internal funding from the University Hospitals of Psychiatry and Psychotherapy and of Child and Adolescent Psychiatry and Psychotherapy, University of Bern and the Soteria Bern.

\section{Author Contributions}

Rahel Flückiger contributed to conceptualization, project administration, investigation and implementation of the group therapy, methodology, data analysis, and visualization. Stefanie J. Schmidt contributed to conceptualization and supervision. Chan- 
tal Michel contributed to project administration and visualization. Jochen Kindler contributed to project administration and supervision. Michael Kaess contributed to project administration, methodology, and supervision. All authors contributed to the preparation of the manuscript.

\section{Data Availability Statement}

All data generated or analysed during this study are included in this published article. The qualitative data generated and analysed during the current study are available from the corresponding author on reasonable request.

\section{References}

1 Hunter ECM, Phillips ML, Chalder T, Sierra M, David AS. Depersonalisation disorder: a cognitive-behavioural conceptualisation. Behav Res Ther. 2003 Dec;41(12):1451-67.

2 American Psychiatric Association, APA. Diagnostic and statistical manual of mental disorders (DSM-5). American Psychiatric Pub; 2013.

3 Hunter EC, Sierra M, David AS. The epidemiology of depersonalisation and derealisation. A systematic review. Soc Psychiatry Psychiatr Epidemiol. 2004 Feb;39(1):9-18.

4 Baker D, Hunter E, Lawrence E, Medford N, Patel M, Senior C, et al. Depersonalisation disorder: clinical features of 204 cases. $\mathrm{Br} \mathrm{J}$ Psychiatry. 2003 May;182(5):428-33.

5 Simeon D. Depersonalisation disorder: a contemporary overview. CNS Drugs. 2004 Aug; 18(6):343-54

6 Hunter EC, Baker D, Phillips ML, Sierra M, David AS. Cognitive-behaviour therapy for depersonalisation disorder: an open study. Behav Res Ther. 2005 Sep;43(9):1121-30.

7 Hunter EC, Charlton J, David AS. Depersonalisation and derealisation: assessment and management. BMJ. 2017 Mar;356:j745.

8 Klosterkötter J, Hellmich M, Steinmeyer EM, Schultze-Lutter F. Diagnosing schizophrenia in the initial prodromal phase. Arch Gen Psychiatry. 2001 Feb;58(2):158-64.

9 Schultze-Lutter F, Debbané M, Theodoridou A, Wood SJ, Raballo A, Michel C, et al. Revisiting the basic symptom concept: toward translating risk symptoms for psychosis into neurobiological targets. Front Psychiatry. 2016 Jan; $7: 9$.

10 Flückiger R, Michel C, Grant P, Ruhrmann S, Vogeley K, Hubl D, et al. The interrelationship between schizotypy, clinical high risk for psychosis and related symptoms: cognitive disturbances matter. Schizophr Res. 2019 Aug;210:188-96.

11 Schultze-Lutter F, Addington J, Ruhrmann S, Klosterkötter J. Schizophrenia proneness instrument adult version (SPI-A). Rome, Italy: Giovanni Fioriti Editore; 2007.

12 Schultze-Lutter F, Marshall M, Koch E. Schizophrenia proneness instrument, child and youth version (SPI-CY), Extended English Translation. Rome, Italy: Giovanni Fioriti Editore; 2012.

13 Schultze-Lutter F, Michel C, Schmidt SJ, Schimmelmann BG, Maric NP, Salokangas $\mathrm{RKR}$, et al. EPA guidance on the early detection of clinical high risk states of psychoses. Eur Psychiatry. 2015 Mar;30(3): 405-16.

14 Hunter EC. Understanding and treating depersonalization disorder. In: Kennedy F, Kennerley $H$, Pearson D, editors. Cognitive behavioural approaches to the understanding and treatment of dissociation. Hove, East Sussex: Routledge; 2013. p. 160-73.

15 Nestler S, Jay EL, Sierra M, David AS. Symptom profiles in depersonalization and anxiety disorders: an analysis of the Beck Anxiety Inventory. Psychopathology. 2015 Mar;48(2): 84-90.

16 Farrelly S, Peters E, Azis M, David A, Hunter EC. A brief CBT intervention for depersonalisation/derealisation in psychosis: study protocol for a feasibility randomised controlled trial. Pilot Feasibility Stud. 2016 Aug; 2(1):47.

17 Michal M. Depersonalisation und Derealisation: die Entfremdung überwinden. 3rd ed. Stuttgart: Kohlhammer Verlag; 2018.

18 Michal M, Adler J, Wiltink J, Reiner I, Tschan R, Wölfling K, et al. A case series of 223 patients with depersonalization-derealization syndrome. BMC Psychiatry. 2016 June;16(1): 203.

19 Simeon D. Depersonalization/depersonalization disorder. In: Gabbard GO, editor. Gabbard's treatments of psychiatric disorders. 5th ed. Washington, DC: APA, American Psychiatric Pub; 2007. p. 459-70.

20 Sierra M, Senior C, Dalton J, McDonough M, Bond A, Phillips ML, et al. Autonomic response in depersonalization disorder. Arch Gen Psychiatry. 2002 Sep;59(9):833-8.

21 Michal M, Koechel A, Canterino M, Adler J, Reiner I, Vossel G, et al. Depersonalization disorder: disconnection of cognitive evaluation from autonomic responses to emotional stimuli. PLoS One. 2013 Sep;8(9):e74331.

22 Hayes SC, Strosahl KD, Wilson KG. Acceptance and commitment therapy. Washington, DC: American Psychological Association; 2009.

23 Neziroglu F, Donnelly K. Dissociation from an acceptance-oriented standpoint. In: Kennedy F, Kennerley H, Pearson D, editors. Cognitive behavioural approaches to the understanding and treatment of dissociation. Hove: Routledge; 2013. p. 236-48.

24 Van der Gaag M, Nieman D, Van den Berg D. CBT for those at risk of a first episode psychosis: evidence-based psychotherapy for people with an "at risk mental state". Routledge; 2013.

25 World Health Organization. The ICD-10 classification of mental and behavioural disorders: clinical descriptions and diagnostic guidelines. Geneva: World Health Organization; 1992.

26 Sierra M, Berrios GE. The cambridge depersonalisation scale: a new instrument for the measurement of depersonalization. Psychiatry Res. 2000 Mar;93(2):153-64. 